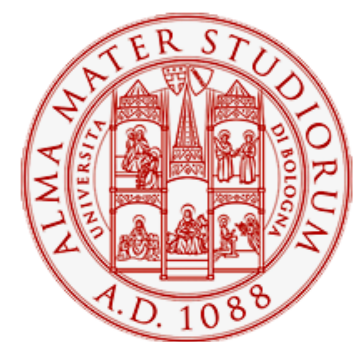

Alma Mater Studiorum - Università di Bologna DEPARTMENT OF ECONOMICS

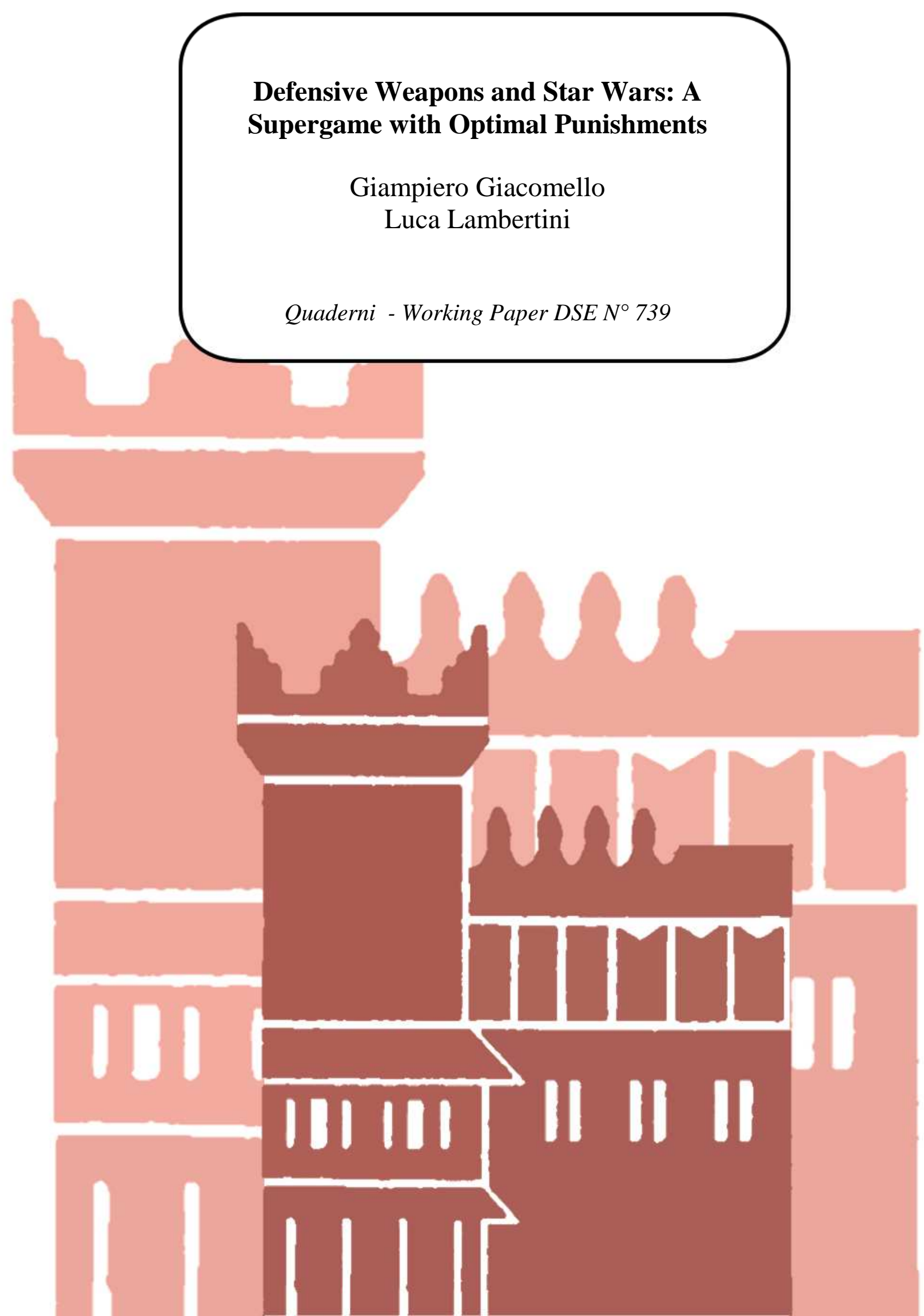




\title{
Defensive Weapons and Star Wars: A Supergame with Optimal Punishments*
}

\author{
Giampiero Giacomello ${ }^{\S}$ and Luca Lambertini $\#$ \\ $\S$ Department of Politics, Institutions, History, University of Bologna \\ giampiero.giacomello@unibo.it \\ \# Department of Economics, University of Bologna \\ luca.lambertini@unibo.it
}

March 11, 2011

\begin{abstract}
We model the perspective faced by nuclear powers involved in a supergame where nuclear deterrence is used to stabilise peace. This setting allows us to investigate the bearings of defensive weapons on the effectiveness of deterrence and peace stability, relying on one-shot optimal punishments. We find that the sustainability of peace is unaffected by defensive shields if both countries have them, while a unilateral endowment of such weapons has destabilising consequences.
\end{abstract}

Keywords: defensive shields, deterrence, wargames

JEL codes: C73, F51

${ }^{*}$ We would like to thank Arsen Palestini and Alessandro Tampieri for useful comments and suggestions. The usual disclaimer applies. 


\section{Introduction}

In a recent paper, Chassang and Padró I Miquel (2009) discuss the role of defensive shields in nuclear conflicts, an issue that has remained a hot one throughout the Cold War and has become even more so in coincidence with the Strategic Defense Initiative (commonly known as 'Star Wars') during the Reagan administration and afterwards. This particular topic nests into a more general debate concerning escalation and deterrence, precisely because defensive shields may ultimately jeopardise the deterrence capability of nuclear arsenals. ${ }^{1}$ Consequently, a specific discussion has been carried out on this aspect, with a striking lack of consensus. Some, like Wilkening (2000) and Lebovic (2002) have focussed on the efficiency of ballistic missile defence, while others have reached opposite conclusions as to the interplay between defensive shields and deterrence: on one side, there are scholars convinced of the destabilising effects of shields (e.g., Brams and Kilgour, 1988; and Miller, 2001); on the other, there are those sustaining the opposite view (e.g., Powell, 1990, 2003; and Lindsay and O'Hanlon, 2001).

Chassang and Padró I Miquel (2009) use a repeated game framework to show that "the unilateral acquisition of defensive weapons raises the stronger country's predatory incentives and reduces the sustainability of peace" ( $p$. 282). To do so, they model the supergame following the route traced by Friedman (1971), whereby trigger strategies imply that a unilateral strike is followed by an everlasting Nash punishment consisting in permanent conflict. This particular component of the supergame - the design of the punishment phase - is indeed critical, as perpetual conflict may not be sustainable if countries have conspicuous endowments of nuclear weapons, whose massive

\footnotetext{
${ }^{1}$ The number of contributions being published in recent years demonstrate that these aspects remain at the core of international politics even after the end of the Cold War (see Powell, 1990; Zagare and Kilgour, 2000; Danilovic, 2002; Freedman, 2004; Zagare, 2004, to mention only a few).
} 
use in the very first phase of the confrontation would very rapidly annihilate contenders altogether, leaving possibly open the way for a retaliation strike but almost surely not for an infinite repetition of the constituent game. ${ }^{2}$ Therefore, we propose here an alternative approach to the construction of the supergame, based on one-shot optimal punishment (Abreu, 1986). This type of punishment, besides being the most efficient one, has the additional advantage - which is particularly convenient when it comes to assessing the effectiveness of deterrence in preventing the emergence of nuclear conflicts - of allowing us to do altogether away with the Nash equilibrium of the constituent game in building up the equilibrium conditions based on the players' intertemporal payoffs.

As in Chassang and Padró I Miquel (2009), we examine a two-country game, whose constituent stage is a prisoners' dilemma. The main results yielded by our setup can be summarised as follows. In the symmetric case where both countries have defensive shields, (i) the stability condition for the sustainability of perpetual peace is completely independent of the shields, while (ii) the one-shot optimal punishment is inversely related to the shield's effectiveness. If instead only one country is endowed with defensive weapons, then indeed the shield has a destabilising effect on the supergame. While the latter result is independent of the punishment scheme, the former is indeed sensitive to it.

The remainder of the paper is organised as follows. The basic model is illustrated in section 2. Section 3 investigates the supergame. Concluding

\footnotetext{
${ }^{2}$ In this respect, it is worth recalling that, during the Cuba crisis, President Kennedy ordered (among other things) to keep aloft the Strategic Air Command bombers armed with nuclear weapons for a gross total of 7000 megatons around the clock. It is hardly credible that an infinitely repeated game could follow the very first stage. Also note that, for several years, General Curtis LeMay was convinced that his strategic bombers could 'bring the enemy back to the stone age in the first six hours', this all-out attack being known as the 'Sunday punch' (for more details on this, see Rhodes, 1995).
} 
remarks are in section 4 .

\section{The setup}

The model can be quickly laid out as follows. Players are two countries, labelled as 1 and 2 , interacting over time $t=0,1,2,3 \ldots \infty$. Country $i$ 's perperiod payoff is

$$
u_{i}=\pi\left(1-a_{j}(1-\sigma)+z a_{i}(1-\sigma)\right)-b a_{i}^{2}-C(\sigma)
$$

$i, j=1,2, j \neq i$. In (1),

- $\pi>0$ is the instantaneous welfare associated with perpetual peace, gross of any costs associated with building up offensive weapons $a_{i}$ and the defensive shield $\sigma$. Given that we are dealing with the analysis of a possible nuclear confrontation, net payoffs are not necessarily positive (indeed they may become strongly negative). Even in a peaceful situation, the investment in a defensive shield may be so costly to cause $\pi<C(\sigma)$.

- The term $1-a_{j}(1-\sigma)+z a_{i}(1-\sigma)$ multiplying $\pi$ determines how much the aforementioned utility is modified by a war where the intensity of attacks are $a_{i}$ and $a_{j}$, respectively, and both countries are endowed with a shield $\sigma$. Note that to be fully effective the shield should have size one. Accordingly, to capture the idea that, reasonably, building up the 'perfect shield' is indeed an unrealistic objective, we may assume the cost function $C(\sigma)$ associated with the R\&D activities and the 
implementation of the defensive shield obeys the following: ${ }^{3}$

$$
C(\sigma)=0 ; \frac{\partial C(\sigma)}{\partial \sigma}>0 ; \frac{\partial^{2} C(\sigma)}{\partial \sigma^{2}}>0 ; \lim _{\sigma \rightarrow 1} C(\sigma)=\infty .
$$

Parameter $z>0$ measures how sensitive country $i$ is to a strike of intensity $a_{i}$ brought to bear against country $j$, as opposed to the damages inflicted by the opponent through an attack of size $a_{j}$. For any $z \in(0,1)$, country $i$ exhibits what we may define as a net damage aversion, in that any advantage generated by its own strike against $j$ is offset, all else equal, by the damage caused by a comparable amount of, say, nuclear bombing borne by $i$ itself. If instead $z>1$, country $i$ is keen on attacking the adversary no matter what (this may reflect the fact that hawks are in control of $i$ 's actions).

- $b a_{i}^{2}$ is the production cost associated with building up the amount of nuclear weapons $a_{i}$, at decreasing returns to scale.

Before delving into the structure and properties of the repeated game, we briefly illustrate the basic features of the constituent one-shot game.

\subsection{The one-shot game}

The equilibrium of the one-shot constituent game can be quickly characterised. The first order condition of country $i$ is:

$$
\frac{\partial u_{i}}{\partial a_{i}}=z(1-\sigma) \pi-2 b a_{i}=0
$$

yielding $a_{i}^{*}=z(1-\sigma) \pi /(2 b)$. For future reference, it is worth noting that (3) implies that the two countries' best reply functions are orthogonal to

${ }^{3}$ For instance, a cost function with these properties is

$$
C(\sigma)=\frac{1}{1-\sigma}-1
$$

This would indeed imply $\pi-C(\sigma)<0$ for values of $\sigma$ sufficiently close to one. 
each other, so that the resulting equilibrium is the intersection of dominant strategies. The associated utility is

$$
u^{*}=\frac{4 b[\pi-C(\sigma)]-z(2-z)(1-\sigma)^{2} \pi^{2}}{4 b}>0
$$

for all

$$
b>\max \left\{0, \frac{z(2-z)(1-\sigma)^{2} \pi^{2}}{4[\pi-C(\sigma)]}\right\} .
$$

To this regard, note that, for all $z \in(0,2)$ any $b>0$ suffices to ensure that $u_{i}^{*}>0$.

There remains to verify that $z \in(0,2)$ suffices to imply that the underlying structure corresponds to a prisoners' dilemma. To see this, observe that the decision whether to attack or not can be evaluated by looking at the following $2 \times 2$ reduced form matrix:

\begin{tabular}{cc|c|c|}
\multicolumn{1}{c}{$P$} & \multicolumn{1}{c}{$A$} \\
\cline { 3 - 4 } 1 & $P$ & $u^{P P} ; u^{P P}$ & $u^{P A} ; u^{A P}$ \\
\cline { 3 - 4 } & $A$ & $u^{A P} ; u^{P A}$ & $u^{*} ; u^{*}$ \\
\cline { 2 - 3 } & &
\end{tabular}

\section{Matrix 1: The one-shot game in reduced form}

Pure strategies $P$ and $A$ stand for remaining peaceful and attacking, respectively. The outcome $(A, A)$ portrays the equilibrium of the game in continuous strategies described above.

The payoff accruing to each player when both choose $P$ is generated by $a_{i}=a_{j}=0$, so that it corresponds to

$$
u^{P P}=\pi-C(\sigma)
$$


with

$$
u^{P P}-u^{*}=\frac{z(2-z)(1-\sigma)^{2} \pi^{2}}{4 b}>0
$$

for all $z \in(0,2)$.

Then, consider the unilateral deviation from the peaceful outcome via the dominant strategy $a_{i}^{*}=z(1-\sigma) \pi /(2 b)$, yielding to the deviator a payoff equal to

$$
u^{A P}=\pi-C(\sigma)+\frac{z^{2}(1-\sigma)^{2} \pi^{2}}{4 b}>0
$$

while the country enduring the strike gets

$$
u^{P A}=\pi-C(\sigma)-\frac{z(1-\sigma)^{2} \pi^{2}}{2 b}
$$

with

$$
u^{A P}-u^{P P}=u^{*}-u^{P A}=\frac{z^{2}(1-\sigma)^{2} \pi^{2}}{4 b}>0 \text { always. }
$$

Hence,

Lemma 1 The one-shot game reflects the structure of a prisoners' dilemma for all $z \in(0,2)$.

As is well known, repeating the prisoners' dilemma over an infinite horizon may allow players to attain Pareto-superior outcomes (as compared to the Nash equilibrium of the one-shot constituent game), provided they are enough forward looking, i.e., their intertemporal preferences must satisfy a condition whose specific nature will depend upon the rules of the supergame itself (in particular, the duration and intensity of the punishment phase). These aspects are investigated in the next section.

\section{The supergame}

The backbone of the literature on supergames is the so called Folk Theorem, that has evolved over the decades taking several alternative forms. From a 
qualitative point of view, the different formulations of this theorem yield the invariant message that infinite repetition allows players to reach any equilibrium outcomes Pareto-dominating that of the one-shot game, provided the players themselves be patient enough. The research efforts carried out over the last four decades have pointed at refining the structure of the punishment phase in order to yield the most efficient outcome, all else equal. For this reason, we will focus here on a supergame based upon Abreu's one-shot optimal punishments (Abreu, 1986). Then, the performance of optimal punishments will also be contrasted with that of the version of this supergame based on an infinite Nash reversion, as in Friedman (1971), although the latter has to be taken with a pinch of salt for the aforementioned reasons.

Countries, being symmetric in all respects, are assumed to have the same time preferences, represented by the time-invariant discount factor $\delta \in[0,1]$. The rules of the supergame unravelling under the deterrence exerted by oneshot optimal punishments can be spelled out as follows.

- At $t=0$, both countries play strategy $P$ (i.e., $a_{i}=0$ ).

- At any $t \geq 1$, both countries keep playing $P$ provided both have played $P$ at $t-1$; otherwise, if any deviation from $(0,0)$ has been observed at $t-1$, then at $t$ countries are to implement the symmetric optimal punishment $a^{o p}$.

- Subsequently, at $t+1$ players return to strategy $P$ provided that both of them have implemented the optimal punishment at $t$. If not (i.e., if at least one of them has deviated from the punishment), they are again required to play $a^{o p}$.

This last rules entails that abiding by the symmetric optimal punishment simultaneously confines the punishment phase to a single period, while any deviation from it extends the punishment phase itself. 
The individual payoff generated by the adoption of symmetric optimal punishments is:

$$
u^{o p}=\pi-C(\sigma)-a^{o p}\left[b a^{o p}+(1-z)(1-\sigma) \pi\right]
$$

while the optimal unilateral deviation from the optimal penal code is given by the dominant strategy $a_{i}^{*}=z(1-\sigma) \pi /(2 b)$, yielding the payoff

$$
u^{D o p}=\pi-C(\sigma)-a^{o p}(1-\sigma) \pi+\frac{z^{2}(1-\sigma)^{2} \pi^{2}}{4 b}>u^{o p} \text { always. }
$$

Perpetual peace is sustainable forever on the basis of the deterrence exerted by optimal punishments if and only if the following conditions are met:

$$
\begin{aligned}
& u^{A P}-u^{P P} \geq \delta\left(u^{P P}-u^{o p}\right) \\
& u^{D o p}-u^{o p} \geq \delta\left(u^{P P}-u^{o p}\right)
\end{aligned}
$$

Inequality (13) has to be met in order for unilateral deviations from the perpetual peace path not to be advantageous, while (14) establishes that deviations from the symmetric optimal punishment are not attractive.

This yields a system of two inequalities in two unknowns, the discount factor $\delta$ and the optimal punishment $a^{o p}$. Solving, we obtain that (13-14) are simultaneously satisfied by any pair

$$
a^{o p} \geq \frac{z(1-\sigma) \pi}{b} ; \delta \geq \frac{z}{4}
$$

This proves the following result:

Lemma 2 Under the threat of optimal punishments $a^{o p} \geq z(1-\sigma) \pi / b$, the two countries can sustain perpetual peace provided their common time preferences satisfy $\delta \geq z / 4$.

A direct consequence of the above lemma is 
Proposition 3 The intensity of the optimal punishment is monotonically decreasing in the effectiveness of the shield $\sigma$. Instead, the stability of perpetual peace in altogether independent of $\sigma$.

As an ancillary observation, it is worth noting that

$$
\frac{z}{4} \in\left[0, \frac{1}{2}\right] \forall z \in[0,2] \text {. }
$$

More interesting is the fact that, the critical level of the discount factor being independent of the presence and size of shields that in turn affects the harshness of the punishment, the foregoing analysis also entails:

Corollary 4 In the absence of shields (i.e., if $\sigma=0$ ), perpetual peace is again sustainable for all $\delta \geq z / 4$. However, the punishment has to reach its peak at $a^{o p} \geq z \pi / b$.

That is, the stability condition is exactly the same but it needs to be accompanied by a threat whose intensity is magnified as much as possible by the absence of any defensive screens whatsoever.

To appreciate the role of optimal punishments in determining the condition for peace stability, we may briefly dwell upon the stability condition generated by the alternative punishment consisting in an infinite reversion to the Nash equilibrium of the constituent game - as it has been used in the supergame analysed by Chassang and Padró I Miquel (2009). Like them, we will look at the admittedly quite unrealistic perspective in which perpetual war after a unilateral first strike is admissible. Following Friedman (1971), the perpetual stability of peace requires

$$
\begin{gathered}
\frac{u^{P P}}{1-\delta} \geq u^{A P}+\frac{\alpha u^{*}}{1-\delta} \\
\Leftrightarrow \frac{z(1-\sigma)^{2} \pi^{2}(2 \delta-z)}{4(1-\delta) b} \geq 0
\end{gathered}
$$


which is met by all $\delta \geq z / 2$. As expected, this of course is higher than (indeed, twice as high as) the critical discount factor generated by optimal punishments. ${ }^{4}$ It is worth stressing that, as is the case under optimal punishments, also the infinite Nash reversion yields a stability condition that is completely independent of defensive paraphernalia.

To this regard, it can be shown that completely different considerations hold true in the more realistic case in which war cannot continue ad infinitum. To see this, assume first that one period of symmetric conflict suffices to erase the two countries. ${ }^{5}$ Peace stability requires:

$$
\frac{u^{P P}}{1-\delta} \geq u^{A P}+\delta u^{*}
$$

In general, if war may be sustained for, say, $T-1$ periods after the first strike, the above inequality rewrites as

$$
\frac{u^{P P}}{1-\delta} \geq u^{A P}+u^{*} \sum_{t=1}^{T} \delta^{t}
$$

with

$$
\sum_{t=1}^{T} \delta^{t}=\frac{1-\delta^{T+1}}{1-\delta}-1=\frac{\delta-\delta^{T+1}}{1-\delta} .
$$

The presence of shields may extend the terminal date $T$ ensuring the survival of both countries over a longer horizon, whereby we may take $T$ as a function of shields $\sigma$ and suppose that $\partial T(\sigma, \sigma) / \partial \sigma>0$. If so, then

$$
\frac{\partial\left(\frac{\delta-\delta^{T(\sigma, \sigma)+1}}{1-\delta}\right)}{\partial \sigma}=-\frac{\delta^{T(\sigma, \sigma)+1} \ln (\delta) \partial T(\sigma, \sigma) / \partial \sigma}{1-\delta}>0
$$

\footnotetext{
${ }^{4}$ One could perform the same exercise using Axelrod's (1981) tit-for-tat strategies, with qualitatively analogous results.

${ }^{5}$ This is a realistic scenario, if one duly takes into account the global consequences of even a limited use of nuclear weapons. See, e.g., Mills et al. (2008).
} 
because $\ln (\delta)<0$ as $\delta$, in general, will be lower than one. Hence, we may expect shields to exert a destabilising effect precisely because they are likely to keep the supergame going after a first strike. This entails that assessing peace sustainability in a supergame relying on Nash punishments is a tricky task, as the answer ultimately depends on whether or not the punishment phase is indeed infinitely long.

The next question is whether an asymmetric situation in which one country is endowed with defensive weapons while the other is not may give rise to instability. This perspective is dealt with in the following section.

\subsection{The asymmetric supergame}

Now suppose country $i$ has a shield of size $\sigma$ while $j$ hasn't any. The corresponding per period utilities are

$$
\begin{gathered}
u_{i}=\pi\left(1-a_{j}(1-\sigma)+z a_{i}\right)-b a_{i}^{2}-C(\sigma) ; \\
u_{j}=\pi\left(1-a_{i}+z a_{j}(1-\sigma)\right)-b a_{j}^{2} .
\end{gathered}
$$

On the basis of (22), we can identify the relevant payoffs for the construction of the incentive compatibility constraints that have to be satisfied here in order for perpetual peace to be stable all over the supergame.

If $a_{i}=a_{j}=0$, we have $u_{i}^{P P}=\pi-C(\sigma)$ and $u_{j}^{P P}=\pi$, while unilateral deviations from the peaceful path yield, respectively:

$$
u_{i}^{A P}=\pi-C(\sigma)+\frac{z^{2} \pi^{2}}{4 b} ; u_{j}^{A P}=\pi+\frac{z^{2}(1-\sigma)^{2} \pi^{2}}{4 b} .
$$

The punishment profits are

$$
\begin{gathered}
u_{i}^{o p}=\pi\left(1-a_{j}^{o p}(1-\sigma)+z a_{i}^{o p}\right)-b\left(a_{i}^{o p}\right)^{2}-C(\sigma) ; \\
u_{j}^{o p}=\pi\left(1-a_{i}^{o p}+z a_{j}^{o p}(1-\sigma)\right)-b\left(a_{j}^{o p}\right)^{2} .
\end{gathered}
$$

where we may expect to observe $a_{i}^{o p} \neq a_{j}^{o p}$ given the asymmetry of the present setup. Likewise, deviations from the optimal penal code yield asymmetric 
payoffs:

$$
\begin{gathered}
u_{i}^{D o p}=\pi-C(\sigma)+\pi\left(\frac{z^{2} \pi}{4 b}-a_{j}^{o p}(1-\sigma)\right) ; \\
u_{j}^{o p}=\frac{\pi\left(z^{2}(1-\sigma)^{2} \pi+4\left(1-a_{i}^{o p}\right) b\right)}{4 b} .
\end{gathered}
$$

The set of constraints to be satisfied for the sustainability of perpetual peace is

$$
\begin{aligned}
& u_{i}^{A P}-u_{i}^{P P} \geq \delta_{\iota}\left(u_{i}^{P P}-u_{i}^{o p}\right) \\
& u_{i}^{D o p}-u_{i}^{o p} \geq \delta_{i}\left(u_{i}^{P P}-u_{i}^{o p}\right)
\end{aligned}
$$

with $i=1,2$. Note that the discount factor is indexed as well since under asymmetric conditions the relative stability thresholds are necessarily asymmetric. Solving these four inequalities, we obtain

$$
\begin{aligned}
\delta_{i} & \geq \delta(\sigma, 0) \equiv \frac{z}{4(1-\sigma)^{2}} ; \delta_{j} \geq \delta(0, \sigma) \equiv \frac{z(1-\sigma)^{2}}{4} ; \\
a_{i}^{o p} & \geq \frac{z \pi}{b} ; a_{j}^{o p} \geq \frac{z(1-\sigma) \pi}{b} .
\end{aligned}
$$

Now note that

$$
\frac{\partial \delta(\sigma, 0)}{\partial \sigma}=\frac{z}{2(1-\sigma)^{3}}>0 \forall \sigma \in[0,1),
$$

whereby we can state:

Proposition 5 Monopoly power on defensive weapons has clearcut destabilising effects on the supergame, as the country being unilaterally endowed with the shield will be more tempted to strike than otherwise.

It is true that $\partial \delta(0, \sigma) / \partial \sigma<0$, so that the other country acts indeed as a puppy $\operatorname{dog},{ }^{6}$ but unfortunately country $i$ faces a more demanding threshold and this may have awful consequences on the stability of peace, as already highlighted by Chassang and Padró I Miquel (2009).

\footnotetext{
${ }^{6}$ This label, identifying a player as a comparatively innocuous domestic pet, dates back to Fudenberg and Tirole (1984).
} 
As an ancillary observation, note that $\delta(\sigma, 0)=\delta(0, \sigma)=z / 4$ if $\sigma=0$ while, for any positive value of $\sigma$, we have $\delta(\sigma, 0)>z / 4>\delta(0, \sigma)$. To sum up our analysis, we may now formulate the following:

Theorem 6 For all $\delta \in[\delta(\sigma, 0), 1]$, perpetual peace is sustainable irrespective of the relative defensive endowments; for all $\delta \in[z / 4, \delta(\sigma, 0))$, perpetual peace is sustainable only if countries have symmetric defensive endowments; for all $\delta \in[0, z / 4)$, perpetual peace is unsustainable.

Now, to complete the picture, we may turn again to the stability requirement generated by the reversion to the one-shot Nash equilibrium à la Friedman (1971) after the first strike has taken place. It suffices to look at the condition faced by the country that has endowed itself with a defensive shield, since if this is violated then necessarily a war will break out. The relevant inequality is defined as in (19), with

$$
\sum_{t=1}^{T(\sigma, 0)} \delta^{t}=\frac{1-\delta^{T(\sigma, 0)+1}}{1-\delta}-1=\frac{\delta-\delta^{T(\sigma, 0)+1}}{1-\delta} .
$$

and

$$
\frac{\partial\left(\frac{\delta-\delta^{T(\sigma, 0)+1}}{1-\delta}\right)}{\partial \sigma}=-\frac{\delta^{T(\sigma, 0)+1} \ln (\delta) \partial T(\sigma, 0) / \partial \sigma}{1-\delta}>0,
$$

which is qualitatively (although not quantitatively) the same as in the fully symmetric setting.

\section{Concluding remarks}

We have revisited the issue of the interplay between deterrence and defensive weapons in a repeated game with optimal one-shot punishments, to show that the sustainability of peace is unaffected by defensive shields if each country has one, while the monopoly of such weapons has indeed destabilising consequences. 


\section{References}

[1] Abreu, D.J. (1986), "Extremal Equilibria of Oligopolistic Supergames", Journal of Economic Theory, 39, 191-225.

[2] Axelrod, R. (1981), "The Emergence of Cooperation among Egoists", American Political Science Review, 75, 306-18.

[3] Brams, S.J. and D.M. Kilgour (1988), "Deterrence versus Defense: A Game-Theoretic Model of Star Wars", International Studies Quarterly, 32, 3-28.

[4] Chassang, S. and G. Padró I Miquel (2009), "Defensive Weapons and Defensive Alliances", American Economic Review, 99 (P\&P), 282-86.

[5] Danilovic, V. (2002), When the Stakes are High: Deterrence and Conflict among Major Powers, Ann Arbor, University of Michigan Press.

[6] Freedman, L. (2004), Deterrence, Cambridge, MA, Polity Press.

[7] Friedman, J.W. (1971), "A Non-Cooperative Equilibrium for Supergames", Review of Economic Studies, 28, 1-12.

[8] Fudenberg, D. and J. Tirole (1984), "The Fat-Cat Effect, the Puppy-Dog Ploy, and the Lean and Hungry Look", American Economic Review, 74, 361-66.

[9] Lebovic, J.H. (2002), "The Law of Small Numbers: Deterrence and National Missile Defense", Journal of Conflict Resolution, 46, 455-83.

[10] Lindsay, J.M. and M.E. O'Hanlon (2001), Defending America: The Case for Limited National Missile Defense, Washington, DC, Brookings Institution Press. 
[11] Miller, S.E. (2001), "The Flawed Case for Missile Defense", Survival, 43, 95-109.

[12] Mills, M.J., O.B. Toon, R.P. Turco, D.E. Kinnison and R.R. Garcia (2008), "Massive Global Ozone Loss Predicted following Regional Nuclear Conflict", Proceedings of the National Academy of Sciences, 105, 5307-12.

[13] Powell, R. (1990), Nuclear Deterrence Theory, Cambridge, Cambridge University Press

[14] Powell, R. (2003), "Nuclear Deterrence Theory, Nuclear Proliferation, and National Missile Defense", International Security, 27, 86-118.

[15] Wilkening, D.A. (2000), "Ballistic-Missile Defense and Strategic Stability", Adelphi Paper no. 334, London, International Institute for Strategic Studies.

[16] Zagare, F.C. (2004), "Reconciling Rationality with Deterrence: A Reexamination of the Logical Foundations of Deterrence Theory", Journal of Theoretical Politics, 16, 107-41.

[17] Zagare, F.C. and D.M. Kilgour (2000), Perfect Deterrence, Cambridge, Cambridge University Press. 


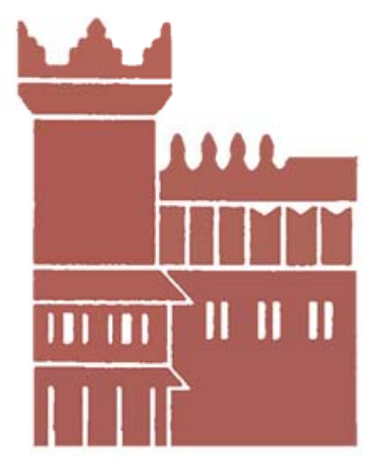

Alma Mater Studiorum - Università di Bologna DEPARTMENT OF ECONOMICS

Strada Maggiore 45

40125 Bologna - Italy

Tel. +39051 2092604

Fax +390512092664

http://www.dse.unibo.it 